\title{
A learning tool for the PHS Policy
}

Researchers applying for grant funding, newly appointed IACUC members and long-time animal care and veterinary personnel who need to brush up on federal policy, read on! The Office of Laboratory Animal Welfare (OLAW), part of the US National Institutes of Health (NIH), has developed an online tutorial on the Public Health Service Policy on Humane Care and Use of Laboratory Animals (PHS Policy). The PHS Policy lists relevant requirements for the care and use of animals in US governmentfunded research. The full text of the PHS Policy, as amended in 2002 , can be found online by following the link from the first paragraph of the tutorial's home page. It is also available for download as a PDF file (http://grants.nih.gov/grants/olaw/references/PHSPolicyLabAnimals.pdf).

The tutorial features a convenient list of contents with direct links to important subpages so that those who know what they are looking for can scan the list and click straight through to their topic of interest. For those who need to learn about the PHS Policy and its requirements, an IACUC's roles and responsibilities or the type of work covered by the PHS Policy, the tutorial provides a full course.

The 'Introduction' section, illustrated by a cartoon of a mouse, includes the document that provides the legal background for the PHS Policy, the Health Research Extension Act of 1985. The section also introduces OLAW and mentions its educational activities. At the time we visited the site, the educational programs included 15 workshops and conferences in 11 cities over the course of a year. The introduction section also lists all of the PHS agencies that support research.

The next section, illustrated with a cartoon of a donkey, goes through the documents related to the PHS Policy: the Guide for the Care and Use of Laboratory Animals; the 9 US Government Principles for the Utilization and Care of Vertebrate Animals Used in Testing, Research, and Training; the Animal Welfare Act and the American Veterinary Medical Association Guidelines for the Euthanasia of Animals.

The 'Terms and Concepts' section, illustrated with a hamster, provides relevant definitions, such as those for a program of animal care and use, the Institutional Official, an Animal Welfare Assurance and enforced self-regulation.

The next section goes deeper into the requirements for a 'Program of Animal Care and Use,' such as the requirement for

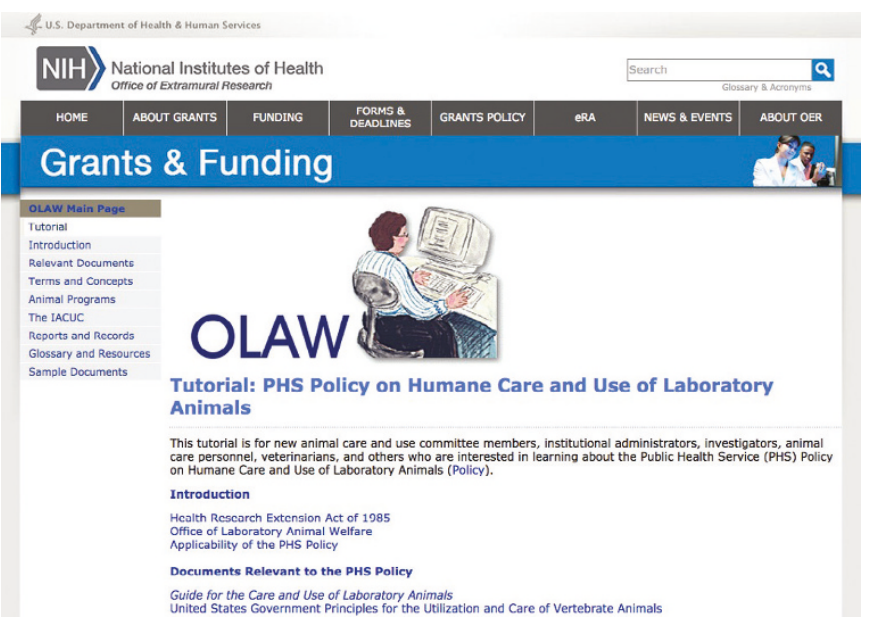

an IACUC, and a brief list of its responsibilities. There's a short definition of 'Veterinary Care;' the requirement for personnel to be qualified and trained; a brief description of the role of an occupational health and safety program for animal care and use; design of facilities and husbandry of animals; and, as we have learned through hurricanes Katrina, Rita and Sandy, the need for disaster planning and emergency preparedness.

Other topics covered include the role of the IACUC, recordkeeping and reports required, a glossary, links to various agencies and laboratory animal organizations, and sample documents.

Even for those who are well acquainted with the rules and workings of an IACUC, there are times that the PHS Policy and its interpretation are unclear. This tutorial leads to additional information that can be helpful, such as in cases of reporting noncompliance or sanctions and suspensions. Each section contains links to other sites and other documents, many of which are more in-depth than the tutorial itself. For example, the OLAW website contains much more material, including updated notices. Readers will want to bookmark this site for easy access.

\section{http://www.grants.nih.gov/grants/olaw/tutorial/ index.htm}

\section{Content}

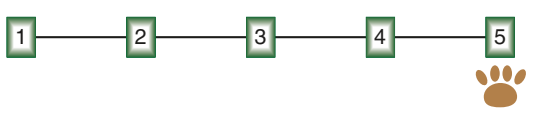

Appearance

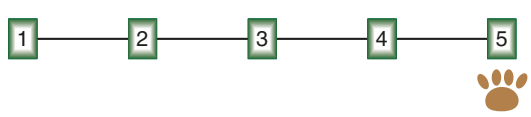

Usability

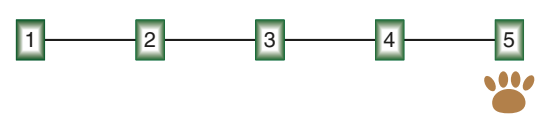

